\title{
Hysteresis in Sawtooth Crash in ASDEX Upgrade Tokamak
}

\author{
O. Dumbrajs ${ }^{*}, 1,2$, V. Igochine ${ }^{3}$, H. Zohm ${ }^{3}$ and ASDEX Upgrade Team ${ }^{3}$ \\ ${ }^{1}$ Helsinki University of Technology, Association Euratom-Tekes, P.O. Box 2200, FIN-02015 HUT, Finland \\ ${ }^{2}$ Institute of Solid State Physics, Association Euratom-University of Latvia, Kengaraga Street 8, LV-1063, Riga, Latvia \\ ${ }^{3}$ MPI für Plasmaphysik, Euratom-Association, D-85748 Garching, Germany
}

\begin{abstract}
A hysteresis model is used to describe experimental data on sawtooth crash in the ASDEX Upgrade tokamak. The model is based on hysteresis which arises due to the fact that the value of the current density gradient (approximated for the H-mode discharges studied here, by the temperature gradient) at the $q=1$ surface required to turn on the instability is greater than the gradient required to maintain the instability, once it is turned on. The value of the hysteresis parameter can be chosen such that the model reproduces correctly the two time scales of the sawtooth crash in the ASDEX Upgrade tokamak: the slow rise time $(\sim 7 \mathrm{~ms})$ and the rapid crash time $(\sim 50 \mu \mathrm{s})$.
\end{abstract}

\section{INTRODUCTION}

Under a wide range of operating conditions tokamaks are subject to a relaxation oscillation in the centre of the plasma. This appears as a sawtooth oscillation of the temperature and density and is also observed on other plasma parameters. During the stable ramp phase heating raises the temperature and at the collapse the associated thermal energy is released to the outer part of the plasma in the form of a heat pulse. Usually it is assumed that the collapse is due to an instability which has an $m=1, n=1$ structure and is associated with the existence of a $q=1$ surface in the plasma. The sawtooth oscillations usually start when a $q=1$ surface first appears. The $q$ profile also oscillates with a sawtooth-like behavior. During the ramp phase the current density around the axis increases and at the collapse it is reduced. Correspondingly the central value of $q$ falls during the ramp and rises at the collapse.

In spite of the huge amount of both the experimental and the theoretical work devoted to studying of this phenomenon (see [1] and references therein), sawtooth oscillations are far from being completely understood.

Recently [2] we investigated non-complete sawtooth reconnection in the ASEDEX Upgrade tokamak. Such reconnection phenomena are associated with internal $m / n=1 / 1$ kink mode which does not vanish after the crash phase (as would be the case for complete reconnection). It was shown that this sawtooth can not be fully described by pure $m / n=1 / 1$ mode and that higher harmonics play an important role during the sawtooth crash phase. Here the Hamiltonian formalism was employed to reconstruct perturbations to model incomplete sawtooth reconnection. It was demonstrated that stochastization appears due to excitation of low-

*Address correspondence to this author at the Institute of Solid State Physics, Association Euratom-University of Latvia, Kengaraga Street 8, LV1063, Riga, Latvia; E-mail: Olgerts.Dumbrajs@lu.lv order resonances which are present in the corresponding $q$ profiles inside the $q=1$ surface which reflects the key role of the $q_{0}$ value. Depending on this value two completely different situations are possible for one and the same mode perturbations: (i) the resonant surfaces are present in $q$ profile leading to stochasticity and sawtooth crash ( $q_{0} \approx 0.7 \pm 0.1$ ); (ii) the resonant surfaces are not present which means no stochasticity in the system and no crash event $\left(q_{0} \approx 0.9 \pm 0.05\right)$. Accordingly central safety factor value is always less than unity in case of non-complete sawtooth reconnection. With such a stochastic model we could well describe experimental observations. In [3] we derived heat diffusion coefficient in a stochastic magnetic field and solved the nonstationary diffusion equation which allowed us to study time evolution of sawtooth crash in ASDEX Upgrade.

In this paper we demonstrate that different timescales in a natural manner can be explained by means of the hysteresis phenomenon. We modify the hysteresis model [4] based on the transport catastrophe. In that model the sawtooth is shown to be independent of the growth of the $m=1$ mode and is related to a catastrophic bifurcation of the anomalous transport coefficient. The magnetic component in fluctuation increases as the plasma pressure increases. If the pressure gradient exceeds a certain threshold, a magnetic stochasticity sets in and the thermal conductivity is enhanced. The increment of the transport associated with the strong magnetic perturbation continues until the pressure profile is flattened. Hysteresis behavior of transport flux to the pressure gradient is obtained. The current profile is influenced by the enhanced current diffusivity from the same mechanism, but the change in the $q$ profile remains small. Different from [4], we use experimentally inferred values for the heat conductivity in the ramp up and the crash phase to model the experimentally observed time scales. A comparison with the theoretically implied values from Ref. [4] is also given. 
Generally speaking various hysteresis phenomena in plasma physics are well known. In relation to sawtooth oscillations one can mention [5] and [6]. In [5] the hysteresis relation between the growth rate of the trigger mode and the gradient of soft X-ray (SX) emissivity at the rational surface was observed at the $q_{52}$ sawtooth crash in Heliotron-E3. In [6] it was found that the value of the sawtooth period in the current ramp-up differs from the value at the same elongation in ramp-down. This hysteresis is attributed to the dependence of the sawtooth period on the electron density which was not kept constant throughout the discharge. In [7] the double hysteresis in $\mathrm{L} / \mathrm{H}$ transition has been examined. It was shown that multiple bifurcations in the hysteresis curve appear. In [8] the bifurcation model for radial electric field was used to study the $\mathrm{L} / \mathrm{H}$ transition in ASDEX Upgrade tokamak. The "back" transition from H-mode to L-mode in DIII-D has been investigated in [9]. Substantial hysteresis was found in the threshold powers. Hysteresis in transport barrier formation was studied in [10]. It was found that internal transport barriers (ITB) require more external heating power to form, than be sustained. This hysteresis provides valuable clues on their underlying physics. Another manifestation of hysteresis arises in connection with the radius of the ITB. It was found that the power needed to form an ITB, increases with its radius. However once an ITB is formed its width can be increased with lower powers than would be needed to form it directly at that radius. Force balance and hysteresis effects during L/H transitions in the H-1 Heliac as the rf heating power was quasi-statically changed was studied in [11]. Another well known effect described in [12] and [13] is the fact that due to hysteresis in $\beta_{N}$ of neoclassical tearing modes (NTM), they persist with reduced heating power. Sometimes hysteresis occurs in the frequency of edge localized modes (ELM) as described in [14]. Limit cycle solutions of a transition model based on an M-mode hysteresis diagram, but allowing for a probabilistic element in the parameters, are presented in [15]. ELM cycles for gradients below the critical one can occur with finite probability. This aspect of the model can explain the stochastic range of ELM frequencies that are seen in experiments. Hysteresis and the catastrophe model for fast magnetic reconnection onset have been proposed in [16]. By applying the hysteresis curve it was demonstrated that the transition from slow to fast reconnection is catastrophic. An interesting hysteresis effect is discussed in [17] in connection of formation and locking of the "slinky mode" in reversed-field pinches. It was shown that the locking/unlocking phenomenon exhibits considerable hysteresis, since the critical mode amplitude for locking is much greater than the critical amplitude for unlocking. Hysteresis in single helicity and quasi-single helicity states in reversed field pinches has been studied in [18]. It was found that there is a large region of quasi-single helicity (QSH) to multiple helicity (MH) hysteresis, i.e. a large range helicity over which both states exist.

Generally all these models are based on the concept of the so-called "hidden variables" (see, for example, [19]) in studying the transition phenomena in plasmas and are related to bifurcations of certain plasma parameters like radial electric field, or temperature gradient used in the present model.

\section{THEORETICAL MODEL}

To describe experimental data on sawtooth crash in ASDEX Upgrade tokamak we adopt the model which has been used in [20] in studying avalanches in continuum driven dissipative systems. We write Eqs. 1a, 1b, and 1c of [20] in the following form:

$$
\begin{aligned}
& \frac{\partial T(r, t)}{\partial t}=\chi(t) \frac{\partial^{2} T(r, t)}{\partial r^{2}}+S(r) \\
& \frac{\partial \chi(t)}{\partial t}=\frac{Q(|\partial T / \partial r|)-\chi(t)}{\tau} \\
& Q(|\partial T / \partial r|)=\left\{\begin{array}{lll}
\chi_{\min }, & \text { low state, } & |\partial T / \partial r|<\beta k \\
\chi_{\max }, & \text { high state } & |\partial T / \partial r|>k
\end{array}\right.
\end{aligned}
$$

Here $T(r, t)$ is the temperature, $\chi(t)$ is the diffusion term, $S(t)$ is the source term, and $\chi_{\max } \gg \chi_{\min }$. The function $Q$ is double valued and dependent upon the history for $\beta k<|\partial T / \partial r|<k$, where $k$ is the instability threshold and $0<\beta<1$. The value of $\mathrm{Q}$ remains in the low state $Q=\chi_{\text {min }}$ until the slope $|\partial T / \partial r|>k$, whereupon it undergoes a transition to the high state. However, when in the high state, $Q=\chi_{\max }$, it will not make the transition to the low state until the slope $|\partial T / \partial r|<\beta k$. Thus $Q$ acts very much like a physical instability in that the value of the slope $k$ required to turn on the instability is greater than the slope $\beta k$ required to maintain the instability once it is turned on (hysteresis!). When $\mathrm{Q}$ changes to $\chi_{\max }$, a magnetic stochasticity sets in and the diffusive term $\chi(t)$ begins to rise toward $\chi_{\max }$.

It should be noted that our model employs the most simple heat conduction equation (1a). This is justified because we wish to study only different time scales in the sawtooth crash disassociating from accompanying changes of plasma parameters.

\section{ASDEX UPGRADE SAWTOOTH DATA}

The experimental temperature profile at ASDEX Upgrade before the sawtooth crash $(\# 20975, \mathrm{t}=4.13 \mathrm{~s})$ can be parameterized as follows:

$T(r / a)=2.46 \cdot\left(1-\left(\frac{r}{a}\right)^{2}\right)^{1.3}$

Here $a=0.5 m$ is the minor radius of the plasma. The $q=1$ surface is located at $r / a=0.3$. This allows us to determine the instability threshold in Eq. (1c): $k=3.6 \mathrm{keV} / \mathrm{m}$. Analyzing sawtooth crash in ASDEX Upgrade by means of the ordinary diffusion equation we found in Ref. 8 that $\chi_{\text {min }} \approx 1 \mathrm{~m}^{2} / \mathrm{s}$ and $\chi_{\text {max }} \approx 2500 \mathrm{~m}^{2} / \mathrm{s}$ and that during the crash time $\tau \approx 40 \mu s$ the central temperature drops to $2.18 \mathrm{keV}$. 
For the source term we choose the following form:

$S(r / a)=S_{0}\left(1-\frac{r / a}{0.3}\right) \quad, r / a \leq 0.3$,

which means that it increases the slope $|\partial T / \partial r|$ linearly.

As a result we have two free parameters in the system of equations (1a-1c): the strength of the source $S_{0}$ and the hysteresis parameter $\beta$. By changing these two parameters we can try to reproduce the experimental number for the full cycle of the sawtooth crash in ASDEX Upgrade which is about $7 \mathrm{~ms}$.

\section{CALCULATIONS}

We impose the Neumann boundary condition at the plasma interior:

$\left.\frac{\partial T}{\partial r}\right|_{r=0}=0$

and the Dirichlet boundary condition

$T\left(\frac{r}{a}=0.30, t\right)=2.18 \mathrm{keV}$

at the position of the $q=1$ surface.

We start the calculations at the moment when the crash is completed, i.e., the temperature profile is flat $(T=2.18 \mathrm{keV})$ and the diffusion coefficient is maximal $\left(\chi=\chi_{\max }\right)$.

The results of the calculations with (3), $S_{0}=240 \mathrm{keV} / \mathrm{s}$ and $\beta=0.50$ are shown in Figs. (1-6). In Figs. $(\mathbf{1}, \mathbf{2})$ one can see characteristic time scales for the instability. The rise time of the temperature is about $0.007 s$ (Fig. 1), while the crash time is about $0.00005 s$ (Fig. 2). In other words, the central temperature (Fig. 3) rises from $2.18 \mathrm{keV}$ up to 2.55 $\mathrm{keV}$ in about $7 \mathrm{~ms}$, but drops from $2.55 \mathrm{keV}$ to $2.18 \mathrm{keV}$ in about $50 \mu \mathrm{s}$. This is related to the behavior of the diffusion coefficient as illustrated in Figs. $(\mathbf{4}, \mathbf{5})$. It appears "suddenly" after about $7 \mathrm{~ms}$ (Fig. 4) and decays to zero in about $150 \mu \mathrm{s}$

(Fig. 5).

In Fig. (6) we illustrate the hysteresis phenomenon as described by the model corresponding to Eq. (1).

By changing the values of the parameters $S_{0}$ and $\beta$ it is possible to regulate the width and the height of sawtooth peaks. Here larger $S_{0}$ values shorten the sawtooth period, but larger $\beta$ values prolong it.

The form and the strength of the source term can be different in different experiments. We have chosen it as (3) only for illustrative purposes. If the source term is taken from experiment, then our model contains only one free parameter $\beta$. After fitting it so that Fig. (1) correctly reproduces experimental observations $(\beta=0.5)$, we can claim that during the crash the temperature gradient at the $q=1$ surface in ASDEX Upgrade drops from $3.6 \mathrm{keV} / \mathrm{m}$ to zero. This is a subject of experimental verification.

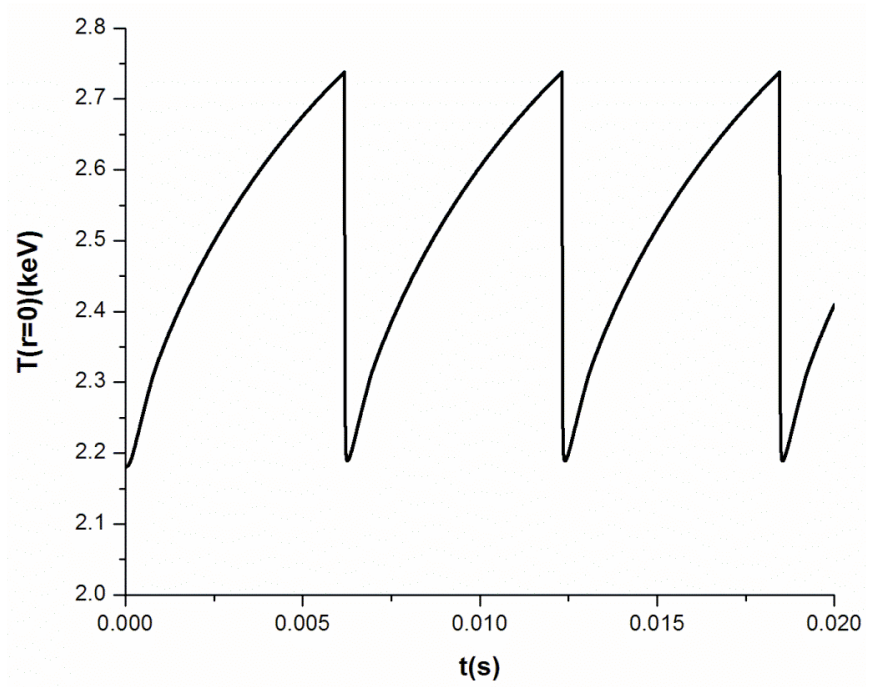

Fig. (1). The periodic crash and build-up time of the central temperature are demonstrated.

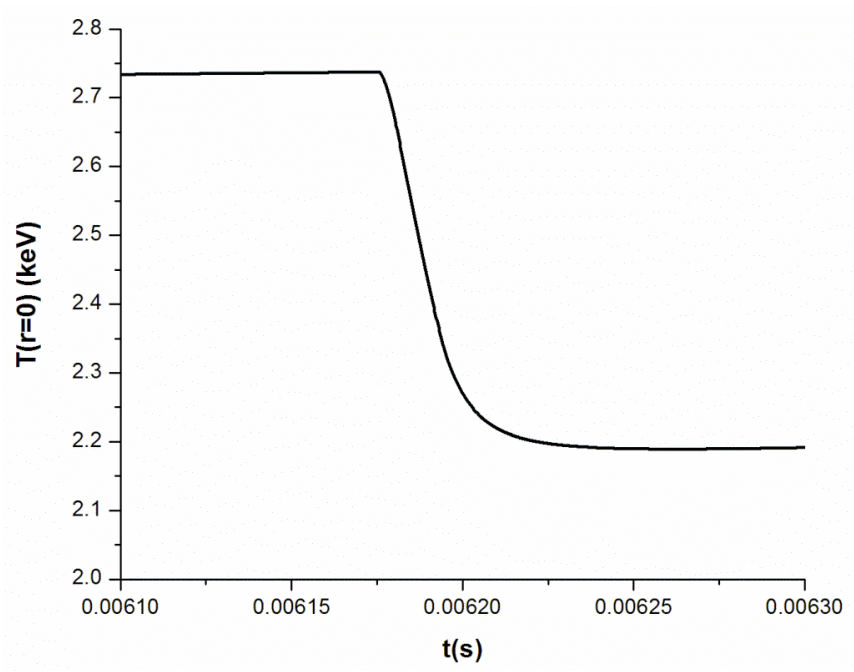

Fig. (2). Temporal dependence of the central temperature in the vicinity of the first peak in Fig. (1).

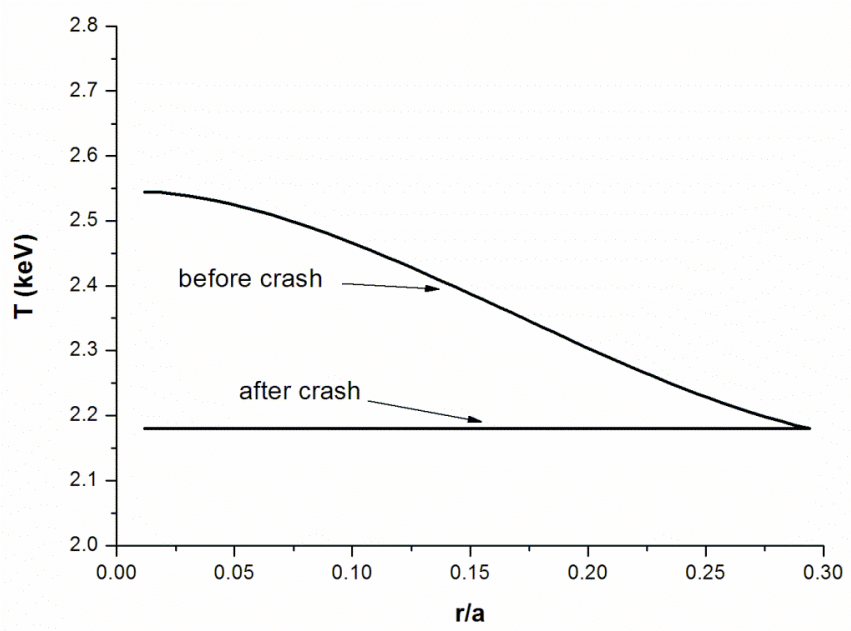

Fig. (3). Temperature profile before and after the crash. 


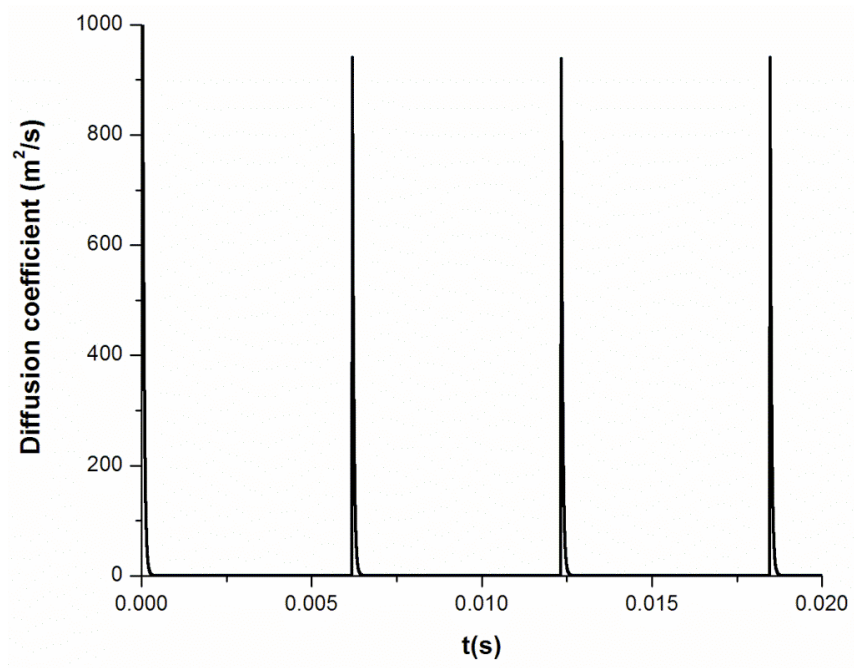

Fig. (4). Temporal evolution of the diffusion coefficient.

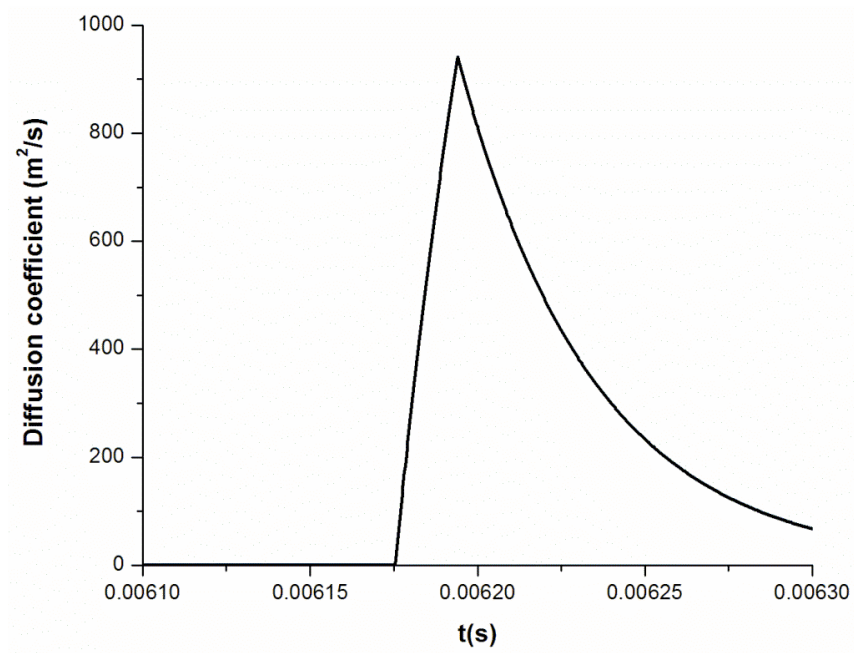

Fig. (5). Temporal dependence of the diffusion coefficient in the vicinity of the first peak.

It is interesting that our results are in a good qualitative agreement with the predictions of the sawtooth model based on the transport catastrophe, as given in [4]. According to that model the crash time of the electron pressure is about few ten times of $\tau_{A p}$, the duration of the burst is a few hundred times $\tau_{A p}$, and the period of the crash and build-up of the pressure gradient is about $1.2 \times 10^{4} \tau_{A p}$, where $\tau_{A p}=a / v_{A p}$. In ASDEX Upgrade $a=0.5 \mathrm{~m}$. Taking into account that the poloidal Alfven velocity

$v_{A p}=\left(\frac{B^{2}}{\mu_{0} n m_{p}}\right)^{1 / 2}$

in ASDEX upgrade for $B=2.379 T$ and $n=6.32^{19} \mathrm{~m}^{-3}$ is $6.5 \cdot 10^{6} \mathrm{~m} / \mathrm{s}$, we obtain $\tau_{A p} \approx 0.07 \mu \mathrm{s}$, which confirms the agreement.

\section{CONCLUSIONS}

A hysteresis model has been used to describe experimental data on sawtooth crash in ASDEX Upgrade tokamak. It is based on hysteresis which arises due to the fact that the value of the temperature gradient at the $q=1$ surface required to turn on the instability is greater than the temperature gradient required to maintain the instability once it is turned on. The value of the hysteresis parameter $\beta$ and the source term have been chosen such that the model reproduces correctly the two time scales of the sawtooth crash in ASDEX Upgrade tokamak: the slow rise time $(\sim 7 \mathrm{~ms})$ and the rapid crash time $(\sim 50 \mu s)$.

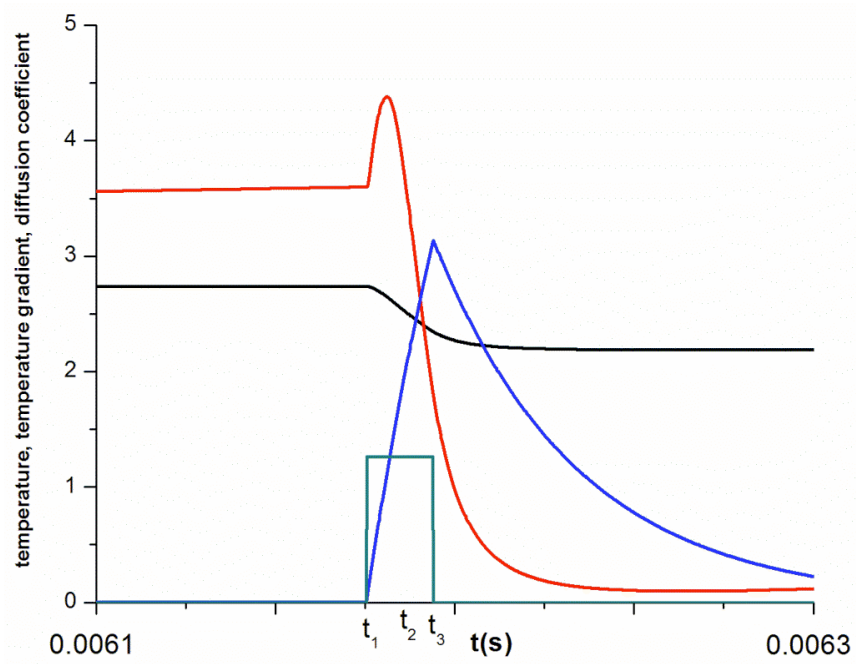

Fig. (6). Hysteresis in sawtooth crash. Temperature gradient (red curve) rises until the instability threshold at $3.6 \mathrm{keV} / \mathrm{m}$. At this moment $t=t_{1}$ the instability is turned on by switching the function $Q$ (green curve, arbitrary units) to $\chi_{\max }$. The central temperature (black curve) begins to decrease and diffusion coefficient (blue curve, arbitrary units) begins to increase. During further evolution due to hysteresis the function $Q$ is switched back to $\chi_{\min }$ not at $t=t_{2}$ when temperature gradient passes again the instability threshold, but at $t=t_{3}$ when temperature gradient becomes equal to $\beta k=1.8 \mathrm{keV} / \mathrm{m}$.

In further studies one should analyze other ASDEX Upgrade discharges, as well as sawtooth discharges in other tokamaks, by means of the proposed model in order to determine "universality" of $\beta$ and $S_{0}$ and to understand the physical nature of $\beta$.

\section{REFERENCES}

[1] Wesson J. Tokamaks. Clarendon Press - Oxford, 2004, pp. 365374, 413-4.

[2] Igochine V, Dumbrajs O, Zohm H, Flaws A, ASDEX Upgrade Team. Stochastic sawtooth reconnection in ASDEX Upgrade. Nucl Fusion 2007; 47: 23-32.

[3] Dumbrajs O, Igochine V, Zohm H, ASDEX Upgrade Team. Diffusion in a stochastic magnetic field in ASDEX Upgrade. Nucl Fusion 2008; 48: 24011-7.

[4] Itoh K, Itoh S-I, Fukuyama A. A sawtooth model based on the transport catastrophe. Plasma Phys Control Fusion1995; 37: 128797.

[5] Takagi S, Toi K, Takechi M, et al. Characteristics of sawtooth oscillations observed in the compact helical system. Phys Plasmas 2004; 11: 1537-44.

[6] Reimerdes H, Furno I, Hofmann F, Martynov A, Pochelon A, Sauter O. Sawtooth behaviour in highly elongated TCV plasmas. Plasma Phys Control Fusion 2006; 48: 1621-32. 
[7] Toda S, Itoh S-I, Yagi M, Fukuyama A, Itoh K. Double hysteresis in $\mathrm{L} / \mathrm{H}$ transition and compound dithers. Plasma Phys Control Fusion 1996; 38: 1337-41.

[8] Zohm H. Dynamic behavior of the $L-H$ transition. Phys Rev Lett 1994; 72: 222-5

[9] Thomas DM, Groebner RJ, Burrell KH, Osborne TH, Carlstrom TN. The back transition and hysteresis effects in DIII-D. Plasma Phys Control Fusion 1998; 40: 707-12.

[10] Annual Report of the EURATOM/UKAEA Fusion Programme 2004/05.

[11] Michael C, Howard J. $13^{\text {th }}$ International Stellarator Workshop, Canberra, Australia 25 Feb-1 Mar 2002.

[12] Zohm H, Gates DA, Wilson HR, et al. Neoclassical MHD in ASDEX Upgrade and COMPASS-D. Plasma Phys Control Fusion 1997; 39: B237-B246.

[13] Maraschek M, Gantenbein G, Goodman TP, et al. $20^{\text {th }}$ IAEA Fusion Energy Conference, Vilamoura, Portugal, 1-6 November 2004, IAEA-CN-116 / EX / 7-2.
[14] Koslowski HR, Nave MFF, Alper B, et al., and contributors to the EFDA-JET workprogramme. $28^{\text {th }}$ EPS Conference on Contr. Fusion and Plasma Phys. Funchal, 18-22 June 2001, ECA, 2001; Vol. 25A: $965-8$.

[15] Itoh S-I, Toda S, Yagi M, Itoh K, Fukuyama A. Physics of collapses: probabilistic occurrence of ELMs and crashes. Plasma Phys Control Fusion 1998; 40: 737-40.

[16] Cassak PA, Shay MA, Drake JF. Catastrophe Model for Fast Magnetic Reconnection Onset. Phys Rev Lett 2005; 95: 235002-6.

[17] Fitzpatrick R. Formation and locking of the "slinky mode" in reversed-field pinches. Phys Plasmas 1999; 6: 1168-93.

[18] Finn JM, Nebel RA, Bathke CG. Single and multiple helicity Ohmic states in reversed-field pinches. Phys Fluids 1992; B4: 1262 79.

[19] Itoh K, Itoh S-I, Fukuyama A. Transport and Structural Formation in Plasmas. Institute of Physics Publishing, Bristol and Philadelphia, 1999, Chapter 16.

[20] Lu ET. Avalanches in Continuum Driven Dissipative Systems. Phys Rev Lett 1995; 74: 2511-4.

(C) Dumbrajs et al.; Licensee Bentham Open.

This is an open access article licensed under the terms of the Creative Commons Attribution Non-Commercial License (http://creativecommons.org/licenses/by$\mathrm{nc} / 3.0 /$ ) which permits unrestricted, non-commercial use, distribution and reproduction in any medium, provided the work is properly cited. 